\title{
A NEW HPLC-UV METHOD COMPARED WITH HPLC-MS FOR DAPTOMYCIN LEVELS IN HUMAN PLASMA SAMPLES
}

Giacomo Luci, Federico Cucchiara, Marianna Lastella, Laura Ciofi, Romano Danesi, Antonello Di Paolo

Department of Clinical and Experimental Medicine, University of Pisa, Pisa, Italy Background Unit of Clinical Pharmacology and Pharmacogenetics, Pisa University Hospital, Pisa, Italy

Daptomycin is the first member of the class of cyclic lipopeptide antibiotic drugs with a broad spectrum of activity against Gram-positive bacteria [1]. The outcome of a therapy with daptomycin in clinical practice is comparable with standard antibiotic treatment and has a linear pharmacokinetics in healthy volunteers with a plasma half-life of about $9 \mathrm{~h}$, with drug excretion via kidneys [2]. However, daptomycin shows additional activity against multi-resistant bacterial strains like methicillin-resistant Staphylococcus aureus (MRSA) [3], vancomycin-resistant enterococci (VRE) [4] or penicillin-resistant Streptococcus pneumoniae [5] and it is therefore a viable alternative for the treatment of persisting infections. Standard doses ranged from 4 to $6 \mathrm{mg} / \mathrm{kg}$, in several cases physicians are prescribing higher dosages up to $10 \mathrm{or} 12 \mathrm{mg} / \mathrm{kg}$ [6]. Its efficacy and safety has been evaluated in a variety of infectious conditions, especially in critical care settings. Some factors may influence daptomycin PK and its PK/PD, especially in hospitalized patients (CKD, GFR $<30 \mathrm{ml} / \mathrm{min}$ ) [7]. Therefore, therapeutic drug monitoring protocols are highly recommended and they can be performed by high performance liquid chromatography (HPLC) systems. Literature and guidelines recommend therapeutic doses for daptomycin [8] to avoid side effects, such as renal failure, hepatotoxicity and rhabdomyolysis [9-10]

\section{Aim}

Application of a new HPLC-UV method on 122 plasma samples of patients from various AOUP wards, after analytical method settings, validation and comparison with a reference LC-MS/MS method. All research activities were performed according to the "Daptolin" protocol (approved by the Pisa University Hospital Ethics Committee, prot. num. 55945)

\section{Instruments, samples and methods}

INSTRUMENTS: The HPLC-UV method was developed using a Waters Alliance 2695 Separations Module equipped with a Waters 2487 Dual $\lambda$ Absorbance Detector (Waters Corporation, Milford, CT) and controlled by the Empower software (version Pro, Waters Corporation).

The LC-MS/MS kit was applied using a Acquity UPLC Binary Solvent Manager pump with Sample Manager autosampler equipped with a TQ Detector (Waters Corporation,). LC-MS/MS system was controlled by MassLynx software (version V4.1, Waters Corporation, USA).

CALIBRATION AND QUALITY CONTROL SAMPLES: A daptomycin stock solution was prepared by dissolving $10 \mathrm{mg}$ of daptomycin in $10 \mathrm{ml}$ of water (final concentration, $1000 \mathrm{mg} / \mathrm{l})$. From this stock solution, $100 \mu \mathrm{l}$ was diluted with $900 \mu \mathrm{l}$ of blank human plasma (obtained from healthy volunteers), obtaining a working solution of $100 \mathrm{mg} / \mathrm{l}$. A gentamicin stock solution was prepared diluting $10 \mu$ of gentamicin $40 \mathrm{mg} / \mathrm{ml}$ in 1 $\mathrm{ml}$ of water (final concentration $400 \mathrm{mg} / \mathrm{l}$ ). From this solution, $100 \mu \mathrm{l}$ were diluted in $900 \mu \mathrm{l}$ of blank human plasma from healthy volunteers, obtaining a working solution of $40 \mathrm{mg} / \mathrm{l}$.

SAMPLE PREPARATION Protein precipitation $=20 \mu \mathrm{l}$ of gentamicin $100 \mathrm{mg} / \mathrm{l}$ such Internal Standard, were added to at $200 \mu \mathrm{l}$ of plasma sample. After vortexing for 30 $\mathrm{sec}$, plasma proteins were precipitated by additing organic solvent. Different tests: Acetonitrile, Methanol, Acetonitrile $+\mathrm{H}_{3} \mathrm{PO}_{4}$ conc. $5 \%(\mathrm{v} / \mathrm{v})$.

HPLC-UV SETTINGS: The HPLC mobile phase consisted of organic solvent (i.e., ACN and/or $\mathrm{MeOH}$ ) plus buffer $\mathrm{KH}_{2} \mathrm{PO}_{4} 20 \mathrm{mM}$ pH=3.2. The choice of final $\mathrm{pH}$ was dependent on the chemical structure of daptomycin and gentamycin in order to optimize the interaction of analytes with stationary phase. Stationary phase: Higgins Analytical C18 $5 \mu \mathrm{m}(250 \mathrm{~mm} \times 4.6 \mathrm{~mm})$ at $35^{\circ} \mathrm{C}$.

Flow: 1,0 ml/min; Injection volume: $50 \mu \mathrm{l}$; UV Wavelenght: $262 \mathrm{~nm}$

LC-MS/MS ANALYSIS: plasma samples analyzed by using a commercial kit (Eureka Lab Division) for LC/MS.

\section{VALIDATION STUDIES [11]}

Linearity range concentrations: daptomycin and gentamicin 5, 10, 25, 50, $100 \mathrm{mg} / \mathrm{l}$; Precision intra-day: 2(1-0.5logConc) $\times 2 / 3$; Inter-day: $2(1-0.5 \log$ Conc)

Specificity was evaluate analyzing interfering peaks at the same retention times of daptomycin and gentamicin. LOD: signal-to-noise ratio $\geq 3$ LOQ: 3.04 x LOD

SAMPLES: From November 2018 to February 2019, 122 plasma samples from AOUP Pisa Hospital wards (Infectious diseases, cardiovascular diseases, orthopaedics, general medicine and neurosurgery) were analyzed.

STATISTICAL ANALYSIS: The software Graph Pad Prism 7 (Graph Pad Software ${ }^{\circledR}$, USA) was used. Correlation analyses were done between daptomycin concentrations of HPLC-UV data and LC-MS/MS reference method. Level of significance was set at $p<0.05$.

\section{Results}

The better recovery after sample analytes extraction was obtained by using ACN $\mathrm{H}_{3} \mathrm{PO}_{4} 85 \%(95: 5 \mathrm{v} / \mathrm{v})$ (Table 1). The mobile phase was chosen on the basis of retention time values and optimal separation, which were achieved by ACN-buffer $\mathrm{KH}_{2} \mathrm{PO}_{4} 20 \mathrm{mM} \mathrm{pH}=3.2$ 46:54, v/v. Retention times were 4.1 and $5.6 \mathrm{~min}$ for daptomycin and gentamicin, respectively (Figures $\mathbf{1}$ and 2). Tables $\mathbf{2}$ and $\mathbf{3}$ report the complete list of analytical parameters for method validation

The performance of the present method was compared with a commercial LC-MS/MS method on 122 human plasma samples. The correlation analysis of measured plasma concentrations returned an $r^{2}$ value of 0.9474 , a slope of 1.052 and a $y$-intercept of $0.8543 \pm 0.9368 \mathrm{mg} / \mathrm{L}$ (Figure 3), without significant differences between the two methods (Mann-Whitney and unpaired t-test with Welch's correction, $p=1.000$ and 0.9927, respectively).

\section{References}

1) Steenbergen et al. J. Antimicrob. Chemother. 2005; 55: 283-288.

2) Dvorchik et al. Antimicrob. Agents Chemother. 2003; 47: 1318-1323.

3) Bassetti et al. Infect. Dis. Complicat. Encount. by Pract. Hematol. 2013; 1: 428-432.

4) Cantón et al. J. Antimicrob. Chemother. 2010; 65: 1126-1136.

5) Carpenter et al. Clin. Infect. Dis. 2004; 38: 994-1000.

6) Bassetti et al. Int. J. Antimicrob. Agents. 2010; 36: 459-461.

7) Dvorchik et al. Antimicrob. Agents Chemother. 2004; 48: 2799-2807

8) Falcone et al. J. Infect. Chemother. 2013; 19: 732-739.

9) Kazory et al. J. Antimicrob. Chemother. 2006; 57: 578-579.

10) Echevarria et al. J. Antimicrob. Chemother. 2005; 55: 599-600.



\section{Conclusions}

In conclusion, a reliable and rapid HPLC-UV method was validated to measure daptomycin concentrations in plasma samples using an internal standard for better accuracy and precision over the range of drug concentrations expected after the administration of daptomycin at standard doses. Furthermore, to our knowledge, this is the first HPLC-UV method for daptomycin that has been compared with a LC-MS/MS reference method. Moreover, the simple preanalytical preparation of samples and the reduced costs of HPLC platform certainly ensure a wide diffusion of the present method, optimal for routine in laboratory. For these reasons, the method is currently used to monitor all plasma samples dispatched to our Clinical Pharmacology Unit.

\section{Contacts}

Dott. Giacomo Luci, ChemPharmD, PhD - e-mail: giacomo.luci@for.unipi.it Prof. Antonello di Paolo, MD, PhD - e-mail: antonello.dipaolo@med.unipi.it

Department of Clinical and Experimental Medicine

University of Pisa

Via Roma 55

56126 Pisa - Italy 